\title{
Dynamic Deployment of Road Side Units for Reliable Connectivity in Internet of Vehicles
}

\author{
Abdulwahab Ali Almazroi, Muhammad Ahsan Qureshi \\ University of Jeddah, College of Computing and Information Technology at Khulais \\ Department of Information Technology \\ Jeddah, Saudi Arabia
}

\begin{abstract}
Internet of vehicles (IoV) promises to provide ubiquitous information exchange among moving vehicles and reliable connectivity to the internet. Therefore, IoV is becoming more and more popular as the number of connected vehicles is increasing. However, the existing vehicular communication infrastructure cannot guarantee reliable connectivity because alltime information exchange for every travelling vehicle is not assured due to lack of required number of roadside units (RSUs) especially along the intercity highways. This study is aimed towards exploring the use of cost-effective dynamic deployment of RSUs based upon the road traffic density and by ensuring the Line of Sight (LOS) among RSUs and Cellular Network Antennas. The unmanned aerial vehicles (UAVs) have a potential to serve as economical dynamic RSUs. Therefore, the use of UAVs along the roadside for providing reliable and ubiquitous information exchange among vehicles is proposed. The UAVs will be deployed along the roadside and their respective placement will be changed dynamically based upon the current traffic density in order to ensure the all-time connectivity with the travelling vehicles and the other UAVs/Cellular Network antennas. The reliability of the proposed network will be tested in terms of signal strength and packet delivery ratio (PDR) using the simulation.
\end{abstract}

Keywords-VANET; Roadside unit; internet of vehicle; social internet of vehicles; unmanned aerial vehicle; line of sight vehicular communication

\section{INTRODUCTION}

Vehicular Ad Hoc Networks (VANETs) [1] provide vehicle to infrastructure (V2I) and vehicle to vehicle (V2V) communication in order to deliver extensive range of applications spanning from infotainment to safety related applications [2, 3]. VANET is gradually evolving into Internet of Vehicles (IoV) that provides internet access to the travelling vehicles in addition to the inter-vehicular communication [4, 5]. The popularity of IoV is increasing rapidly due to its huge potential in terms of connectivity and a wide range of advantageous applications [6]. The modern vehicular communication also offers accident detection [7]. The communication in VANETs depends on the Roadside Unit (RSU) [8]. However, this massive connectivity requires optimal deployment of communication infrastructure; in which roadside units (RSUs) are important component. RSUs provide the essential link among the vehicles and other infrastructure components to fulfill the connectivity challenges of IoV [9]. RSUs connect the vehicles to the other vehicles which are outside their radio range and also with the infrastructure components in order to provide internet access to the vehicles on the road [10].

A comprehensive strategy for installation of RSUs is proposed in [10]. In [11], RSUs are placed by utilizing geometrical methods to improve the LOS among communicating vehicle in modern road infrastructure units. Another scheme introduces deployment of RSUs efficiently in modern roads using approximation algorithm [12]. A similar approach is used in [13]. Most of the existing studies [14-16] focus on the optimized static placement of RSUs by using innovative algorithms to assist vehicular communication. On the other hand, the recent advancements in unmanned aerial vehicles (UAVs) technology can assist the vehicular communication by solving the problem of ubiquitous internet connectivity and all-time inter-vehicle communication. Therefore, a few recent studies $[17,18]$ tend to exploit the use of UAVs (drones)[19]as relays to assist vehicular communication where the road traffic is dense by improving the performance and reducing the network delay. UAVs are becoming efficient, lightweight and economical gradually [19, 20]. A few studies advocate the use of UAVs to assist vehicular communication; however, the focus is to improve the performance and reduce the network delay in dense environment [21]. However, the notion of modern IoV requires ubiquitous internet connectivity and all-time inter-vehicle communication especially when traveling on highways where the road traffic density is sparse; therefore, internet connectivity and vehicular communication are compromised especially in highway environment [12]. Therefore, the existing studies lack in the exploitation of UAVs to serve as potential dynamic RSU especially for the highway (sparse) environment as the RSUs have limited converge area that results in significant degradation in communication. Currently, static placement of RSUs is in practice. The placement of a RSU can be changed accordingly with change in the road traffic density by ensuring the Line of Sight (LOS) among RSUs and Cellular Network Antennas to overcome the limited coverage area issue of RSUs.

The goal of this study is to address the problem of all-time inter-vehicle communication and ubiquitous internet connectivity. Therefore, the aim of current work is to investigate the use of cost-effective dynamic deployment of RSUs based upon the road traffic density and by ensuring the Line of Sight (LOS) among RSUs and Cellular Network Antennas. The study targets the UAVs for economical dynamic deployment of RSUs due to their efficient, lightweight and 
economical nature. Therefore, the use of UAVs along the roadside for providing reliable and ubiquitous information exchange among vehicles is proposed. Consequently, the current study proposes dynamic deployment and placement of UAVs along the roadside based upon the current traffic density. As a result, the proposed method ensures all-time connectivity with the travelling vehicles and the other UAVs/Cellular Network antennas.

To achieve the goal of the study, the objectives of the research are as follows:

Objective 1: To study state-of-the-art in the domain of deployment of RSUs.

Objective 2: To propose dynamic deployment of RSUs based upon road traffic density using UAVs.

Objective 3: To evaluate the proposed solution in terms of connectivity index.

Objective 4: To compare the proposed framework with and without dynamic deployments of RSUs existing solution(s).

The current study follows a research design consisting of five main steps to achieve the objectives of the work. In the first step, a review of state-of-the-art studies in roadside unit deployment is performed. Step 2 focuses on comparison of existing energy efficiency techniques. Developing a cost effective dynamic RSU deployment strategy based on efficient utilization of UAVs is the target of step 3. Evaluation of the proposed solution and comparison of proposed solution with the existing solutions are part of step 4 and 5 .

The rest of the paper is organized as follows: Section 2 presents state-of-the-art studies in roadside unit deployment. This is then followed by Section 3, which describes cost effective dynamic RSU deployment strategy. The evaluation of proposed strategy is explained in Section 4. Section 5 provides a comparison between proposed strategy and existing work. The research implications of the study are highlighted in Section 6 and finally, Section 7 concludes the paper.

\section{RELATED WORK}

Multiple studies were conducted in the past for the efficient deployment of RSUs in VANETs and IoVs. This subsection reviews a few important studies in the domain of RSUs deployment.

Some research utilized artificial intelligence technology to address various problems of IoV such as energy management, traffic monitoring and management, resource management, big data processing, and communication problem [8, 22, 23]. In [24], the authors used reinforcement learning approach called centralized Q-learning for energy efficiency and optimization in IoV. Similarly, [25]fuzzy quality of service is utilized for optimization of energy in IoV. The author in [22] focused on multi-media communication in IoV. On the other hand, UAVs technology has shown enormous potential to provide efficient solutions to diverse problems belonging to different fields of life due to its cost effective, lightweight and efficient nature. Currently, UAVs have wide range of applications in different domains like smart cities, traffic management, military, smart agriculture, smart healthcare, smart houses and industry [26-
29]. In smart cities, UAVs are providing many services such as traffic management, environmental monitoring, pollution monitoring, and security control [27]. The solutions provided by UAVs to traffic management include [28] that proposes a system for smart traffic monitoring to overcome limitations of existing system. Due to the recent advancements in UAVs and the cost-effective nature of UAVs, the study aims to utilize UAVs for providing all-time inter-vehicle communication and ubiquitous internet connectivity by dynamic deployment of RSUs based upon the road traffic density and by ensuring the Line of Sight (LOS) among RSUs and Cellular Network Antennas.

A mechanism to maintain line of sight (LOS) among the travelling vehicles was proposed in [11]. The study considered modern road infrastructures such as flyovers, underpass, curved roads and tunnels. The study utilizes geometrical concepts for the efficient deployment of RSUs, signal enhancers and signal reflectors to maintain LOS among the travelling vehicles. The results of the study were promising as this concept provides reliable connectivity due to the maintenance of LOS among travelling vehicles. However, this study does not consider the most important highway environment where the connectivity is compromised due to coarse density of vehicles.

A cooperative architecture for Intelligent Transportation System (ITS) [30] based upon distributed RSUs was presented in [31]. Using this architecture, the data from all the sensors was collected in a distributed fashion without the intervention of a central control system. It defines the role of each individual network element in the controlling the sensors and disseminating the information. Real-world experiments were also conducted to prove the correctness of idea. However, this study does not focus on the reliability issues of information dissemination as no discussion is presented on the efficient deployment of RSUs.

In an attempt to identify the optimal number of RSUs in a highway environment, a research was conducted that analyzed the delay of message dissemination in VANETs [32]. Based upon the analysis result, the RSUs were deployed at optimal distances from each other. The experimental results also verify optimal RSUs placement. However, this study does not consider the dynamic RSU deployment in VANET environment.

Another attempt was carried out in order to optimally deploy the RSUs using a novel concept of Minimal Mobility Pattern Coverage (MPC) [14]. Firstly, the mobility of travelling vehicle is predicted from a trace file. Secondly, based upon the extracted information, the optimal placement of RSUs is advocated by extracting minimal traversal of a hypergraph. The authors claim that the experiment results validate the proposed research. However, this study is yet not verified at large scale. Furthermore, the deployment of RSUs is based upon predicted mobility pattern. Therefore any change in the mobility pattern will negatively affect the optimal placement of RSUs.

In a relatively recent study [33], an algorithmic approach towards optimal placement of one-dimensional RSU is presented. A strategy called “dynamic limiting” is first used for 
pruning the search space. A greedy algorithm named "OptDynLim" is proposed that optimally identifies the placement of RSU. The results of the study are formally verified and validated with the help of simulations. However, this study only considers signal dimension RSU deployment problem and don not focuses on general RSU deployment.

Another study [34] exists in the literature that focuses collectively on the 2-D RSU deployment and service task assignment in the domain of IoV. A linear programming based clustering algorithm was proposed that considers the delay requirements and the task assignment. The comparison of the algorithmic output with the optimal solution proved the workability of the proposed solution. However, this study lacks in considering the changing traffic density requirement and dynamic deployment of RSUs.

A study [35] presented a state-of-the-art review of the applicability of UAVs in modern transportation system in smart city environment. This study has highlighted the potential uses of UAVs in ITS and the challenges that may encountered during the implementation. The potential applications include the accident reporting and police eye, along with the flying road side unit. Therefore, this study can also serve as a pivotal point towards the dynamic RSU deployment.

\section{PRoposed FrAMEWORK}

In this section, the proposed solution is discussed that provide efficient, light weight, and cost effective method to provide all-time inter-vehicle communication and ubiquitous internet connectivity by dynamic deployment of RSUs based on UAVs. To achieve the objective of the work, first a mathematical model is formulated, then based on the proposed mathematical model, an extensive simulation is carried out to obtained the result. The proposed solution place RSUs dynamically based upon the road traffic density and by ensuring the Line of Sight (LOS) among RSUs and Cellular Network Antennas. In the proposed solution, a road is divided into $n$ segments and a road segment has a specific number of poles. Mostly, these poles are part of infrastructure. One UAV is assigned to every road segment. Based on the traffic density, the UAVs containing RSU can change its location dynamically from one pole to another in the same road segment. This solution is mathematically represented below:

The mathematical model of proposed solution is described below:

Let $t \in P$ where $p=\left\{p_{1}, p_{2} \ldots p_{n}\right\}$

Here p represents poles in a road segment $\mathrm{R}$.

Let $D=\left\{d_{1}, d_{2}, \ldots d_{m}\right\}_{\text {where } m<n}$

Here, D describes the number of drones. It is to be noted that the number of drones is less than the number of poles in a road segment $\mathrm{R}$.

$R=\left\{\left(p_{1}, p_{2}\right),\left(p_{2}, p_{3}\right), \ldots\left(p_{n-1}, p_{n}\right)\right\}$
$C_{i}$ represents number of vehicles in any $\mathrm{R}$ at time $T i$ and $d_{T}$ is the density threshold. For no change in the scenario, $C_{i}<d_{T}$

$D=\forall d \in D$ there exists $\exists p \in P \mid(d, p)$

Otherwise,

$D=\forall d_{i} \in D$ there exists $\exists P_{i} \in P \mid\left(d_{i}, p_{i+1}\right) C_{i} \geq d_{T}$

At time $\mathrm{T}_{1}$, D may have following values depicting that which particular drone is present at which pole at the moment.

$D=\left\{\left(d_{1}, p_{1}\right),\left(d_{2}, p_{4}\right), \ldots\left(d_{m}, p_{n-1}\right)\right\}$

\section{EXPERIMENTAL SETUP}

In the current research, three scenarios are selected for performing experimental results. The details of these scenarios are presented in Table I. For the scenario 1 and 2, M2 motorway of Pakistan, and Makkah-Madinah highway are selected, respectively. Khulais, urban area of Makkah province of Saudi Arabia is selected for urban scenario. The topologies of roads selected in our scenarios are imported to SUMO simulator by utilizing OpenStreetMap utility. A total of three dynamic RSUs are deployed with a range of one $\mathrm{Km}$. The minimum and maximum vehicles for highway scenario 1 and 2 are same, which are, 1 and 50, respectively. However, due the different nature of urban scenario the minimum number of vehicles is 10 and the maximum number of vehicles is 60 . Further, minimum and maximum communicating vehicles are same for highway scenario while urban scenario has different minimum and maximum vehicles as depicted in Table I. For both highway scenarios a three KM segment of road is selected. On the other hand, nine square KM segment is preferred for ubran scenario. Fig. 1 depicted the highway scenario 1, i.e. M2 motorway of Pakistan. Urban scenario in SUMO is portrayed in Fig. 2 and Fig. 3 shows the urban scenario with running vehicles in SUMO.

TABLE I. DETAIL OF EXPERIMENTAL SETUP

\begin{tabular}{|c|c|c|c|c|c|c|}
\hline $\begin{array}{l}\text { Scena } \\
\text { rios }\end{array}$ & $\begin{array}{l}\text { Minim } \\
\text { um } \\
\text { Vehicl } \\
\text { es }\end{array}$ & $\begin{array}{l}\text { Maxi } \\
\text { mum } \\
\text { Vehicl } \\
\text { es }\end{array}$ & $\begin{array}{l}\text { Minimum } \\
\text { Communi } \\
\text { cating } \\
\text { Vehicles }\end{array}$ & $\begin{array}{l}\text { Maximum } \\
\text { Communi } \\
\text { cating } \\
\text { Vehicles }\end{array}$ & Area & $\begin{array}{l}\text { Selecte } \\
\text { d } \\
\text { Highw } \\
\text { ays }\end{array}$ \\
\hline $\begin{array}{l}\text { Highw } \\
\text { ay } \\
\text { Scenar } \\
\text { io } 1\end{array}$ & 1 & 50 & 1 & 14 & $\begin{array}{l}3 \mathrm{KM} \\
\text { Segm } \\
\text { ent }\end{array}$ & $\begin{array}{l}\text { M2 } \\
\text { Motor } \\
\text { way, } \\
\text { Pakista } \\
\text { n }\end{array}$ \\
\hline $\begin{array}{l}\text { Highw } \\
\text { ay } \\
\text { Scenar } \\
\text { io } 2\end{array}$ & 1 & 50 & 1 & 14 & $\begin{array}{l}3 \mathrm{KM} \\
\text { Segm } \\
\text { ent }\end{array}$ & $\begin{array}{l}\text { Makka } \\
\text { h- } \\
\text { Madin } \\
\text { a } \\
\text { Highw } \\
\text { ay, } \\
\text { Saudi } \\
\text { Arabia }\end{array}$ \\
\hline $\begin{array}{l}\text { Urban } \\
\text { Scenar } \\
\text { io }\end{array}$ & 10 & 60 & 4 & 17 & $\begin{array}{l}9 \\
\text { Squar } \\
\text { e KM }\end{array}$ & $\begin{array}{l}\text { Khulai } \\
\text { s, } \\
\text { Makka } \\
\text { h, } \\
\text { Saudi } \\
\text { Arabia }\end{array}$ \\
\hline
\end{tabular}




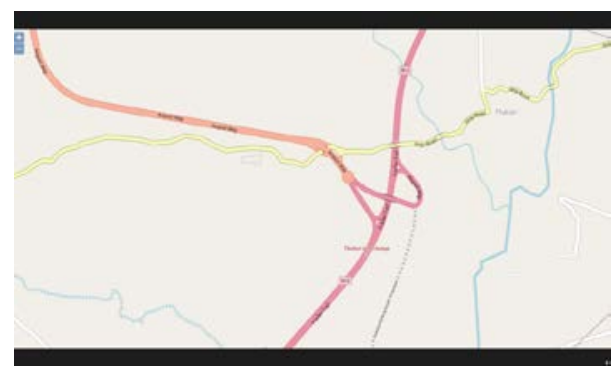

Fig. 1. High Way Scenario.

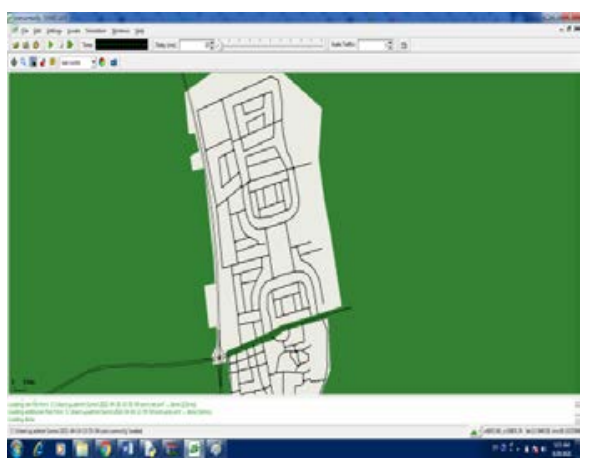

Fig. 2. Urban Scenario in SUMO.

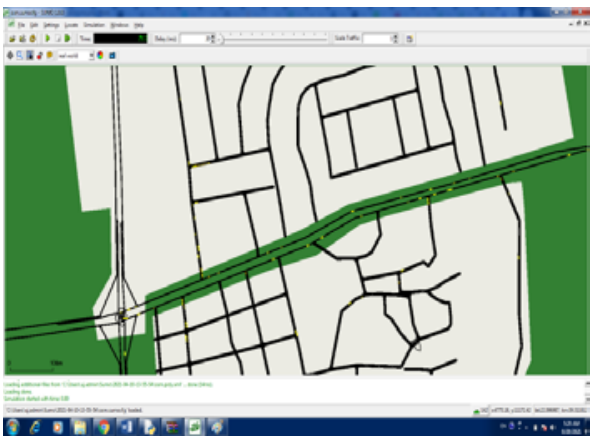

Fig. 3. Urban Scenario with Running Vehicles.

\section{EXPERIMENTAL RESULTS}

This section presents the results of connectivity index and percentage index for three selected scenarios.

Fig. 4, 5 and 6 described the connectivity index with dynamic deployment of RSUs for scenario 1, 2, and 3 respectively. The connectivity index elevates with the increased number of vehicles, however, important point to note here is, due to dynamic deployment, the connectivity index has raised $19 \%$ and $25 \%$, respectively. Consequently, it is inferred that, with the deployment of RSUs dynamically, significant improvement in connectivity index is achieved resulting in enhanced vehicular communication. Correspondingly, connectivity index upgraded $13 \%$ in urban scenario supporting the previous inference. However, the boost in vehicular communication in urban scenario is less as compared to highway scenarios. Hence, it is concluded that the proposed dynamic deployment is more suitable to the highway scenarios as compared to urban scenario. Further, extensive research is required for the dynamic deployment of RSUs in urban scenario to take maximum advantage of the proposed deployment.

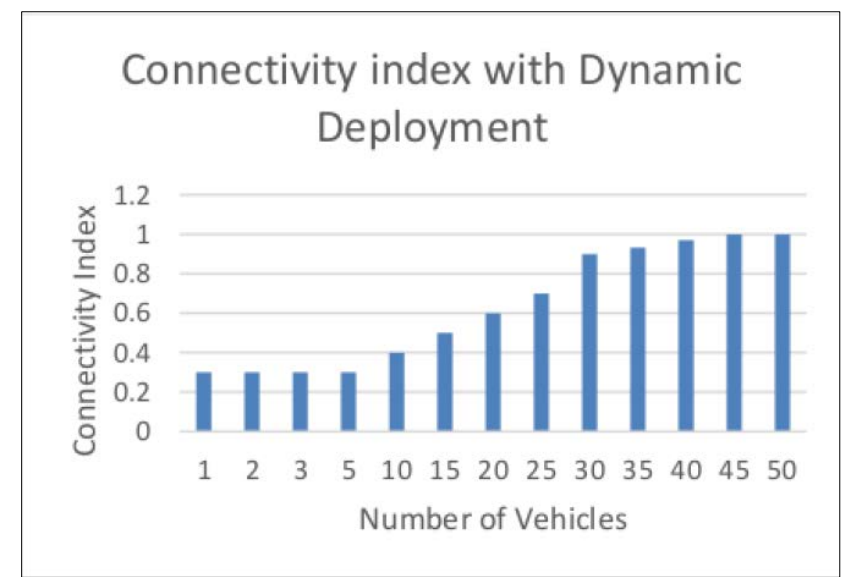

Fig. 4. Connectivity Index for Highway Scenario 1.

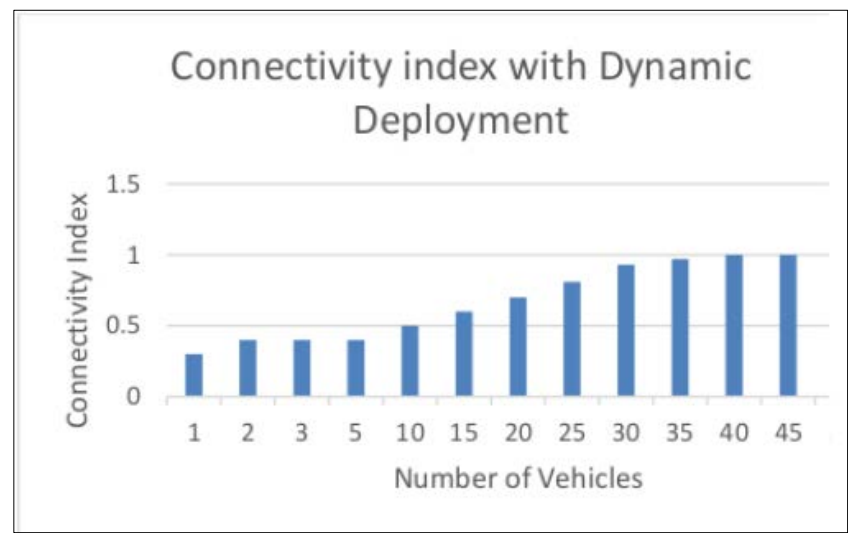

Fig. 5. Connectivity Index for Highway Scenario 2.

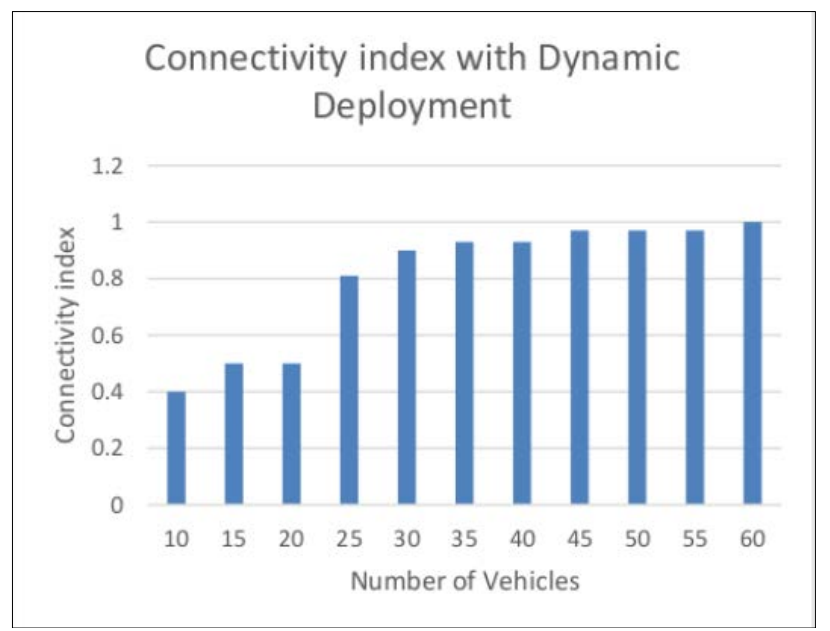

Fig. 6. Connectivity Index for Urban Scenario.

Fig. 7 exhibited the amplification in vehicular communication due to dynamic deployments of RSUs. A considerable escalation is attained in the all scenarios. For highway scenario 1 and 2, gain in connectivity is $19 \%$ and $25 \%$, respectively. On the other hand, the gain connectivity is $13 \%$. These results support the dynamic deployment of RSUs in highway scenarios as well as in urban scenarios. However, in urban scenarios, the connectivity gain can be enhanced by conducting further research as discussed earlier. 


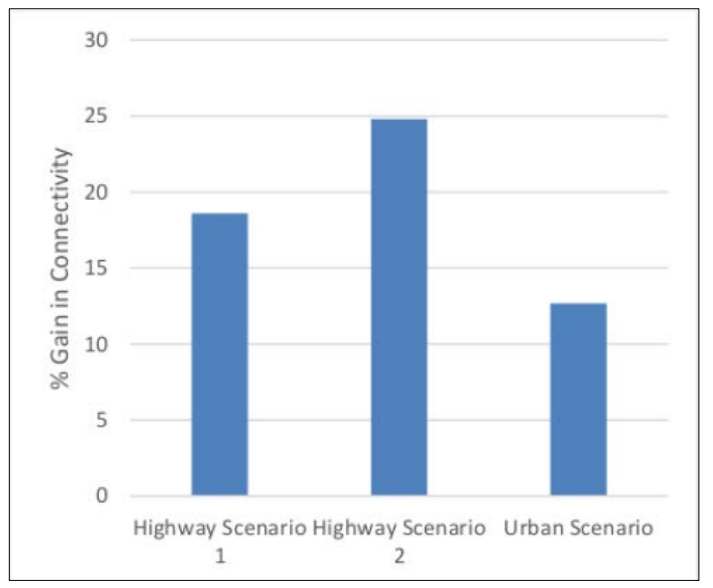

Fig. 7. \% Gain in Connectivity of all Scenarios.

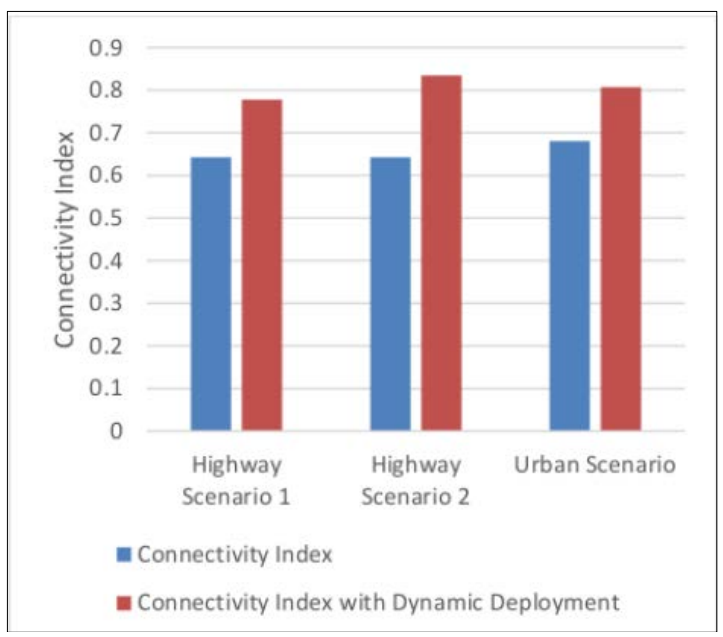

Fig. 8. Comparison of Connectivity Index with and without Dynamic Deployment for all Scenarios.

It is also important to validate the results to show the improvement in connectivity index due the proposed dynamic deployment of RSUs. For this purpose, the current work compares the connectivity index with and without dynamic deployment of RSUs for all scenarios. Fig. 8 illustrates this comparison. A superior connectivity index has achieved in all scenarios due to the deployment of RSUs dynamically. The highway scenario 2 obtained the highest connectivity index while the lowest connectivity index is associated with highway scenario 1 with the proposed method.

\section{RESEARCH IMPLICATIONS}

The findings of the studies can be beneficial to for scientific community. The findings can be utilized to design and develop new standards, applications, and protocols on the basis of the dynamic RSU deployment. It will provide a cost effective solution to the classic RSU deployment problem. Further, the implications of the findings of the study are twofold in scientific community perceptive; (i) the new standards, applications, and protocols in $\mathrm{IoV}$ and modern transportation domains will be evaluated considering the maximum reliability in message dissemination, and (ii) the cost deployment and maintenance of RSUs will be reduced. In the society betterment prospective, the results can be exploited in multiple manners. In line with the vision 2030 of Saudi Arabia, the cost effective solution in modern transportation environment along the highways will be beneficial for the general public and transportation companies in terms of reliability of information exchange during the travel. Specially, the expected outcomes are advantageous for huge traffic movement during the Hajj and Umrah seasons.

\section{CONCLUSION, FUTURE WORK AND LIMITATIONS}

Currently, IoV provides Internet connectivity between moving vehicles; however, all-time connectivity among moving vehicles is not assured due to the limited number of RSUs. Consequently, busy intercity highways are facing a problem of limited connectivity to the Internet resulting in reduced information exchange. To address this limitation, the current study proposes, mathematically modeled, experimented, and evaluated a scheme to dynamically place RSUs in highways and urban scenarios. The placement of RSUs is based on road traffic density by ensuring line of sight among RSU and Cellular Network Antennas. The proposed scheme is evaluated based on connectivity index that describes the communication among vehicles. The experimental results for three scenarios: highway scenario 1 , highway scenario 2 , and urban scenario highlighted the superiority of proposed scheme in terms of connectivity index. The connectivity index is considerably higher in the proposed scheme for all of the scenarios resulting in increased vehicular communication. Thus, addressing the problem of vehicular communication in IoV. The study has some limitations too. First, the proposed scheme is evaluated on small scale with three scenarios. Second, for the large scale evaluation, only simulation results are presented. Third, real-world evaluation is not executed. Therefore, future directions of this work include real-world evaluation of the scheme. Moreover, more scenarios from different geographical region will be select to evaluate the proposed scheme.

\section{ACKNOWLEDGMENT}

This work was funded by the Deanship of Scientific Research (DSR), University of Jeddah, Jeddah, under grant No. (UJ-20-093-DR)The authors, therefore, acknowledge with thanks DSR technical and financial support.

\section{REFERENCES}

[1] M. A. Qureshi, R. M. Noor, S. Shamshirband, S. Parveen, M. Shiraz, and A. Gani, "A survey on obstacle modeling patterns in radio propagation models for vehicular ad hoc networks," Arabian Journal for Science and Engineering, vol. 40, pp. 1385-1407, 2015.

[2] H. Ye, G. Y. Li, and B.-H. F. Juang, "Deep reinforcement learning based resource allocation for V2V communications," IEEE Transactions on Vehicular Technology, vol. 68, pp. 3163-3173, 2019.

[3] M. Ahsan Qureshi, E. Mostajeran, R. M. Noor, A. Shamim, and C.-H. Ke, "A Computationally Inexpensive Radio Propagation Model for Vehicular Communication on Flyovers and Inside Underpasses," KSII Transactions on Internet and Information Systems (TIIS), vol. 10, pp. 4123-4144, 2016.

[4] O. Kaiwartya, A. H. Abdullah, Y. Cao, A. Altameem, M. Prasad, C.-T. Lin, et al., "Internet of vehicles: Motivation, layered architecture, network model, challenges, and future aspects," IEEE Access, vol. 4, pp. 53565373, 2016.

[5] A. A. Almazroi and M. A. Qureshi, "An Energy-aware Facilitation Framework for Scalable Social Internet of Vehicles," International Journal of Advanced Computer Science and Applications, 2021. 
[6] J. Contreras-Castillo, S. Zeadally, and J. A. Guerrero-Ibañez, "Internet of vehicles: architecture, protocols, and security," IEEE Internet of Things Journal, vol. 5, pp. 3701-3709, 2017.

[7] M. H. Alkinani, A. A. Almazroi, N. Z. Jhanjhi, and N. A. Khan, "5G and IoT Based Reporting and Accident Detection (RAD) System to Deliver First Aid Box Using Unmanned Aerial Vehicle," Sensors (Basel, Switzerland), vol. 21, 2021.

[8] E. S. Ali, M. K. Hasan, R. Hassan, R. A. Saeed, M. B. Hassan, S. Islam, et al., "Machine Learning Technologies for Secure Vehicular Communication in Internet of Vehicles: Recent Advances and Applications," Security and Communication Networks, vol. 2021, 2021.

[9] A. Ali, N. Ayub, M. Shiraz, N. Ullah, A. Gani, and M. A. Qureshi, "Traffic Efficiency Models for Urban Traffic Management Using Mobile Crowd Sensing: A Survey," Sustainability, vol. 13, p. 13068, 2021.

[10] D. Kim, Y. Velasco, W. Wang, R. Uma, R. Hussain, and S. Lee, "A new comprehensive RSU installation strategy for cost-efficient VANET deployment," IEEE Transactions on Vehicular Technology, vol. 66, pp. 4200-4211, 2016.

[11] M. A. Qureshi and R. M. Noor, "Towards improving vehicular communication in modern vehicular environment," in 2013 11th International Conference on Frontiers of Information Technology, 2013, pp. 177-182.

[12] M. Ge and Y. Chung, "Efficient Deployment of RSUs in Smart Highway Environment," International journal of advanced smart convergence, vol. 8, pp. 179-187, 2019.

[13] Z. Wang, J. Zheng, Y. Wu, and N. Mitton, "A centrality-based RSU deployment approach for vehicular ad hoc networks," in 2017 IEEE International Conference on Communications (ICC), 2017, pp. 1-5.

[14] T. Yeferny and S. Allani, "Mpc: A rsus deployment strategy for vanet," International Journal of Communication Systems, vol. 31, p. e3712, 2018.

[15] Z. Gao, D. Chen, S. Cai, and H.-C. Wu, "Optimal and greedy algorithms for the one-dimensional rsu deployment problem with new model," IEEE Transactions on Vehicular Technology, vol. 67, pp. 7643-7657, 2018.

[16] M. F. Faraj, J. F. Sarubbi, C. M. Silva, and F. V. Martins, "A Memetic Algorithm Approach to Deploy RSU s Based on the Gamma Deployment Metric," in 2018 IEEE Congress on Evolutionary Computation (CEC), 2018, pp. 1-8.

[17] Z. Shafiq, R. Abbas, M. H. Zafar, and M. Basheri, "Analysis and Evaluation of Random Access Transmission for UAV-Assisted Vehicular-to-Infrastructure Communications," IEEE Access, vol. 7, pp. 12427-12440, 2019.

[18] M. Khabbaz, J. Antoun, and C. Assi, "Modeling and Performance Analysis of UAV-Assisted Vehicular Networks," IEEE Transactions on Vehicular Technology, vol. 68, pp. 8384-8396, 2019.

[19] Y. Zeng and R. Zhang, "Energy-efficient UAV communication with trajectory optimization," IEEE Transactions on Wireless Communications, vol. 16, pp. 3747-3760, 2017.

[20] G. Jang, J. Kim, J.-K. Yu, H.-J. Kim, Y. Kim, D.-W. Kim, et al., "CostEffective Unmanned Aerial Vehicle (UAV) Platform for Field Plant Breeding Application," Remote Sensing, vol. 12, p. 998, 2020.
[21] N. A. Khan, N. Z. Jhanjhi, S. N. Brohi, A. A. Almazroi, and A. A. Almazroi, "A Secure Communication Protocol for Unmanned Aerial Vehicles," Cmc-computers Materials \& Continua, vol. 70, pp. 601-618, 2022.

[22] A. H. Sodhro, Z. Luo, G. H. Sodhro, M. Muzamal, J. J. Rodrigues, and V. H. C. De Albuquerque, "Artificial Intelligence based QoS optimization for multimedia communication in IoV systems," Future Generation Computer Systems, vol. 95, pp. 667-680, 2019.

[23] M. B. Hassan, E. S. Ali, R. A. Mokhtar, R. A. Saeed, and B. S. Chaudhari, "NB-IoT: concepts, applications, and deployment challenges," in LPWAN Technologies for IoT and M2M Applications, ed: Elsevier, 2020, pp. 119-144.

[24] H. Park and Y. Lim, "Reinforcement learning for energy optimization with $5 \mathrm{~g}$ communications in vehicular social networks," Sensors, vol. 20, p. 2361, 2020.

[25] S. Hu, H. Fan, Z. Wang, and Z. Cai, "A Fuzzy QoS Optimization Method with Energy Efficiency for the Internet of Vehicles," Advances in Networks, vol. 4, p. 34, 2016.

[26] P. K. R. Maddikunta, S. Hakak, M. Alazab, S. Bhattacharya, T. R. Gadekallu, W. Z. Khan, et al., "Unmanned aerial vehicles in smart agriculture: Applications, requirements, and challenges," IEEE Sensors Journal, 2021.

[27] N. Mohamed, J. Al-Jaroodi, I. Jawhar, A. Idries, and F. Mohammed, "Unmanned aerial vehicles applications in future smart cities," Technological Forecasting and Social Change, vol. 153, p. 119293, 2020.

[28] N. A. Khan, N. Jhanjhi, S. N. Brohi, R. S. A. Usmani, and A. Nayyar, "Smart traffic monitoring system using unmanned aerial vehicles (UAVs)," Computer Communications, vol. 157, pp. 434-443, 2020.

[29] F. Syed, S. K. Gupta, S. Hamood Alsamhi, M. Rashid, and X. Liu, "A survey on recent optimal techniques for securing unmanned aerial vehicles applications," Transactions on Emerging Telecommunications Technologies, p. e4133, 2020.

[30] G. Dimitrakopoulos and P. Demestichas, "Intelligent transportation systems," IEEE Vehicular Technology Magazine, vol. 5, pp. 77-84, 2010.

[31] A. Moreno, E. Osaba, E. Onieva, A. Perallos, G. Iovino, and P. Fernández, "Design and Field Experimentation of a Cooperative ITS Architecture Based on Distributed RSUs," Sensors, vol. 16, p. 1147, 2016.

[32] C. Liu, H. Huang, and H. Du, "Optimal RSUs deployment with delay bound along highways in VANET," Journal of Combinatorial Optimization, vol. 33, pp. 1168-1182, 2017.

[33] Z. Gao, D. Chen, S. Cai, and H.-C. Wu, "Optdynlim: An optimal algorithm for the one-dimensional rsu deployment problem with nonuniform profit density," IEEE Transactions on Industrial Informatics, vol. 15, pp. 1052-1061, 2018.

[34] Y. Ni, J. He, L. Cai, J. Pan, and Y. Bo, "Joint Roadside Unit Deployment and Service Task Assignment for Internet of Vehicles (IoV)," IEEE Internet of Things Journal, vol. 6, pp. 3271-3283, 2018.

[35] H. Menouar, I. Guvenc, K. Akkaya, A. S. Uluagac, A. Kadri, and A. Tuncer, "UAV-enabled intelligent transportation systems for the smart city: Applications and challenges," IEEE Communications Magazine, vol. 55, pp. 22-28, 2017. 